\title{
Arrays of holes fabricated by electron-beam lithography combined with image reversal process using nickel pulse reversal plating
}

Yousef Awad, Eric Lavallée, Kien Mun Lau, Jacques Beauvais, Dominique Drouin, Melanie Cloutier, David Turcotte, Pan Yang, and Prasad Kelkar

Citation: Journal of Vacuum Science \& Technology A: Vacuum, Surfaces, and Films 22, 1040 (2004); doi: 10.1116/1.1647589

View online: http://dx.doi.org/10.1116/1.1647589

View Table of Contents: http://avs.scitation.org/toc/jva/22/3

Published by the American Vacuum Society

\section{HDDEN}

\section{Instruments for Advanced Science}

Contact Hiden Analytical for further details: W www.HidenAnalytical.com E info@hiden.co.uk

CLICK TO VIEW our product catalogue

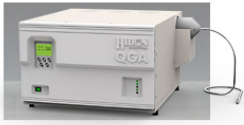

Gas Analysis

dynamic measurement of reaction gas streams catalysis and thermal analysis

- molecular beam studies

dissolved species probes

fermentation, environmental and ecological studies

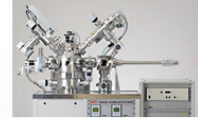

Surface Science , UHVTPD

SIMS

end point detection in ion beam etch elemental imaging - surface mappin

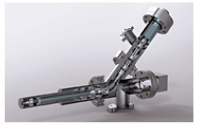

Plasma Diagnostics plasma source characterization etch and deposition process reaction kinetic studies analysis of neutral and radical species

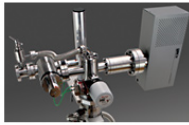

Vacuum Analysis partial pressure measurement and contro of process gases reactive sputter process contro , vacuum diagnostics vacuum coating process monitoring 


\title{
Arrays of holes fabricated by electron-beam lithography combined with image reversal process using nickel pulse reversal plating
}

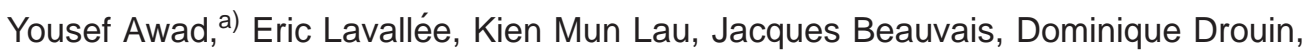 \\ Melanie Cloutier, David Turcotte, Pan Yang, and Prasad Kelkar \\ Quantiscript Inc., 2500 Boulevard Universite, Sherbrooke, Quebec J1K 2R1, Canada
}

(Received 3 December 2003; accepted 15 December 2003; published 18 May 2004)

\begin{abstract}
A critical issue in fabricating arrays of holes is to achieve high-aspect-ratio structures. Formation of ordered arrays of nanoholes in silicon nitride was investigated by the use of ultrathin hard etch mask formed by nickel pulse reversal plating to invert the tonality of a dry e-beam resist patterned by e-beam lithography. Ni plating was carried out using a commercial plating solution based on nickel sulfamate salt without organic additives. Reactive ion etching using $\mathrm{SF}_{6} / \mathrm{CH}_{4}$ was found to be very effective for pattern transfer to silicon nitride. Holes array of $100 \mathrm{~nm}$ diam, $270 \mathrm{~nm}$ period, and 400 $\mathrm{nm}$ depth was fabricated on a $5 \times 5 \mathrm{~mm}^{2}$ area. (c) 2004 American Vacuum Society.
\end{abstract}

[DOI: $10.1116 / 1.1647589]$

\section{INTRODUCTION}

Hole arrays fabricated in GaAs have been used in application for photonic bandgap waveguides. ${ }^{1}$ Ebbesen et al. ${ }^{2}$ showed that hole arrays can be used for extraordinary optical transmission through sub-wavelength holes. Periodic structure of holes can also be used for the purpose of designing new guiding microwave structures. ${ }^{3}$ The reversal imprinting method has been used recently as an alternative to conventional nanoimprint lithography. ${ }^{4}$ This method offers an advantage over conventional nanoimprinting by allowing imprinting onto substrates that cannot be easily spin coated, such as flexible polymer substrates. Nanoindentation using scanning probe microscope $\mathrm{e}^{5}$ has been used to fabricate arrays of high-aspect-ratio holes. Fernandez et al. ${ }^{6}$ studied the use of interference lithography for large-scale production of holes array. This approach, however, suffers from hard-toachieve exposure repeatability, besides maintaining good uniformity of the patterns, which requires perfectly aligned beams. Other researchers ${ }^{7}$ modified the shape of the incident intensity distribution to fabricate arrays of holes, by interfering more than two beams. Although many different configurations of multiple beams can increase image contrast, only those that satisfy certain symmetry requirements preserve the large depth of field characteristic of two beams. To make fine hole patterns, application of optical lithographic techniques such as utilizing an attenuating phase shift mask is used to enhance the resolution, ${ }^{8}$ however, the diameter formed by this approach is restricted to $0.20 \mu \mathrm{m}$ using the $\mathrm{KrF}$ excimer laser. Nakao et al. ${ }^{9}$ have used phase shift masks using $\mathrm{KrF}$ excimer laser as exposure light to fabricate $0.1 \mu \mathrm{m}$ dense holes.

In this article, a simple fabrication method for a nanoimprint lithography mold is demonstrated. This method consists of four major processing steps: stack deposition, e-beam lithography, image reversal, and etching. As it was demonstrated by Alkaisi et al. ${ }^{10}$ silicon nitride as a mold material for nanoimprint lithography presents several advantages in-

${ }^{\text {a)} E l e c t r o n i c ~ m a i l: ~ a w a d @ q u a n t i s c r i p t . c o m ~}$ cluding nonsticking problems between the mold and the used polymer, which may eliminate the need for a release agent or surfactant to overcome the sticking problems. Furthermore, performing the nanoimprint lithography at low temperatures and low pressure could be easily achieved using silicon nitride as a mold material. In this article, sub- $100 \mathrm{~nm}$ patterning is performed using a negative dry e-beam resist. Dry resist is a desirable alternative to wet resist, because pinhole free, good adhesion, uniform and ultrathin film deposition can be achieved easily. Furthermore, dry resists can be deposited on a three-dimensional surface, ${ }^{11}$ or on a very small surface like on tip of monomode fiber. ${ }^{12,13}$ The processing of dry resists has attracted considerable attention in recent years. Many researchers used plasma deposition ${ }^{14,15}$ sublimation or thermal evaporation ${ }^{12}$ to apply dry e-beam resist on a substrate.

To obtain an array of holes, image reversal is performed by plating a thin Ni layer around the exposed resist structures, converting the resist structure into an opening suitable for conventional reactive ion etching. The final step was to use conventional reactive ion etching to ash the resist and to etch through the opening into the underlying layers.

\section{EXPERIMENT}

Figure 1 shows the hole array fabrication process flow used in this work. The fabrication of an array of nanoscale high-aspect-ratio holes consists of four major steps: substrate preparation, patterning, electroplating, and conventional reactive ion etching (RIE).

In stack preparation, a $300 \mathrm{~nm}$ thick $\mathrm{Si}_{3} \mathrm{~N}_{4}$ was deposited by LPCVD on a $365 \mu \mathrm{m}$ thick [100] $\mathrm{Si}$ substrate. The LPCVD $\mathrm{Si}_{3} \mathrm{~N}_{4}$ layer is deposited under light tensile stress, which is believed to reduce the mechanical strain on the nano-scale pillars on the final mold. A thin $(20 \mathrm{~nm})$ silver plating seed layer is deposited on the silicon nitride coated wafer by electron gun evaporation. Silver was chosen as the seed layer for its excellent electrical conductivity and it can be easily dry etched using the same dry etch gas mixture that is being used to etch the underlying $\mathrm{Si}_{3} \mathrm{~N}_{4}$ layer. A $30 \mathrm{~nm}$ 
$30 \mathrm{~nm}$ e-beam resist $-20 \mathrm{~nm} \mathrm{Ag}$ seed layer $300 \mathrm{~nm} \mathrm{Sin}_{4}$ layer Si substrate

\section{a) Stack preparation}

믄

b) Patterning by Electron beam lithography

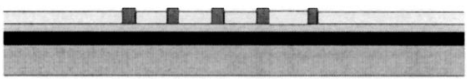

c) Pulse reversal plating of $25 \mathrm{~nm}$ nickel

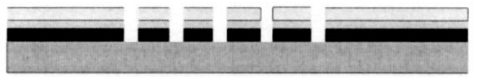

\section{d) Reactive ion etching of resist, $\mathrm{Ag}$ and} $\mathrm{Si}_{3} \mathrm{~N}_{4}$

\section{e) Wet etch of $\mathrm{Ni}, \mathrm{Ag}$}

FIG. 1. Process steps for the fabrication of hole arrays.

thick layer of QSR-5, ${ }^{16}$ a negative dry electron-beam resist which consists mainly of nonpolymeric sterol molecules, is then evaporated on top of the seed layer.

In patterning the substrate, an array of dots was formed in the negative resist by using electron-beam lithography. Patterning the resist was performed using a LEO 1530 field emission gun scanning electron microscope (FEG-SEM) externally controlled by the Nabity Pattern Generation System 9.0. The electron source for this system is a field emission gun (FEG) which makes it possible to maintain a beam diameter better than $5 \mathrm{~nm}$ even at low energy $(<15 \mathrm{keV})$ and at a beam current of $900 \mathrm{pA}$. The patterning was done at 10 $\mathrm{keV}$ energy. In order to reduce the processing time for the pattern containing arrays of dots, a single point exposure scheme was used for each point. This was performed by stepping the beam by $270 \mathrm{~nm}$ increments to fill large squares fields $(200 \times 200) \mu \mathrm{m}^{2}$. The overall exposure dose in the fields is $700 \mu \mathrm{C} / \mathrm{cm}^{2}$, which yields to a point dose of 0.5 $\mathrm{pC} /$ point. After exposure, development of the resist was carried out by immersion in a methyl ethyl ketone (MEK) bath for $10 \mathrm{~s}$, followed by water rinse and then dried using $\mathrm{N}_{2}$.

An image reversal process using Ni pulse reversal plating was used to reverse the tonality of the pattern and to form a hard-etch mask for RIE. Ni electrodeposition was carried out using a commercial plating solution of nickel sulfamate salt without organic additives. Pulse reversal technique was used in this study to improve via/trench filling capability, and to enhance the dissolution of unwanted grains. For the plating of a 20-30 nm nickel film, precise control of the electrodeposition is critical, since the plating rate from the commercial nickel sulfamate bath used in previous works ${ }^{17-19}$ was $\sim 10 \mathrm{~nm} / \mathrm{s}$, using such a plating rate makes the deposi-

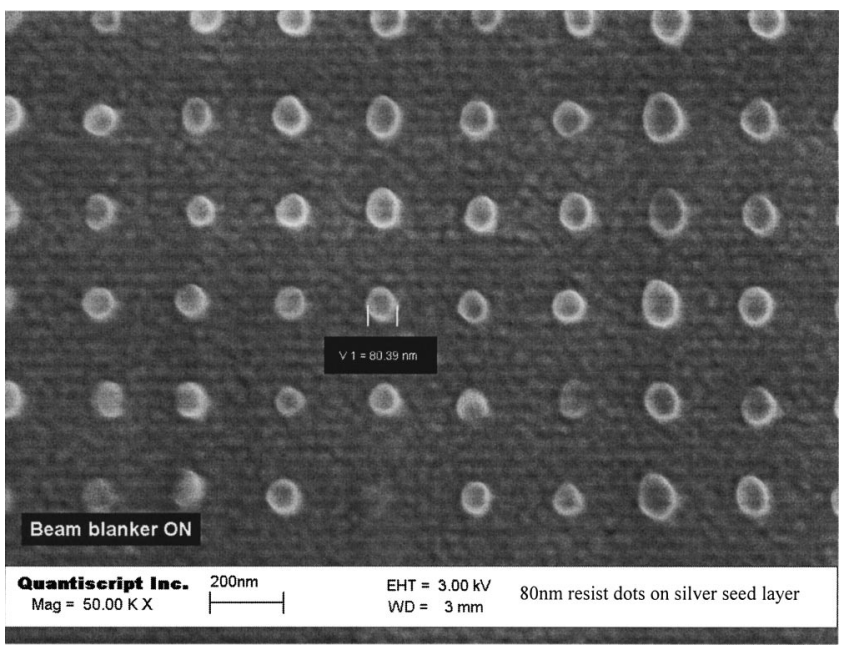

FIG. 2. Array of $100 \mathrm{~nm}$ diam dots patterned in $30 \mathrm{~nm}$ thick dry resist (QSR-5).

tion of 20-30 nm thin film extremely hard to achieve. Furthermore, grain refinement, porosity improvement, and minimization of overplating are essential issues when plating around a resist structure with a $30 \mathrm{~nm}$ thickness.

The plating parameters: average current density, duty cycle, plating frequency, bath temperature, and agitation, were studied and optimized to produce extremely finegrained (i.e., nanocrystalline) deposits free from voids or defects at a very slow plating rate as discussed in the following section. The plated thin film has to be free from voids or defects in order to protect the underlaying substrate during dry etching. It is important to note that stripping the resist patterns, dry etching of $\mathrm{Ag}$ film and subsequently the $\mathrm{Si}_{3} \mathrm{~N}_{4}$ layer were carried out in one etching step in a March 1701 RIE system using a $\mathrm{SF}_{6}: \mathrm{CH}_{4}$ mixture at a power of $100 \mathrm{~W}$ for $30 \mathrm{~min}$. After eching through the holes, the Ni etch mask as well as the silver seed layer were stripped off using concentrated nitric acid.

\section{RESULTS AND DISCUSSION}

Combining e-beam lithography with image reversal and conventional reactive ion etching offers the prospect of patterning arrays of holes with the desired aspect ratio.

Figure 2 shows a SEM of an average $80 \mathrm{~nm}$ diam, $30 \mathrm{~nm}$ height dots fabricated in the negative resist. The smoothest surface and the smallest grain size have been achieved by combining a current reversal plating at $400 \mathrm{kHz}$ frequency, $80 \%$ duty cycle, ultrasonic bath having a power of $80 \mathrm{~W}$, bath temperature of $45^{\circ} \mathrm{C}$, and $1.5 \mathrm{~mA} / \mathrm{cm}^{2}$ average current density. A plating rate as low as $0.2 \mathrm{~nm} / \mathrm{s}$ is obtained using these conditions. This yields a deposit with a mirror smooth finish without any visible grains or holes. Since the plating rate is extremely low, very accurate control of the film thickness is obtained. Figure 3 shows a scanning electron microscopy (SEM) of high resolution image of arrays of holes sur- 


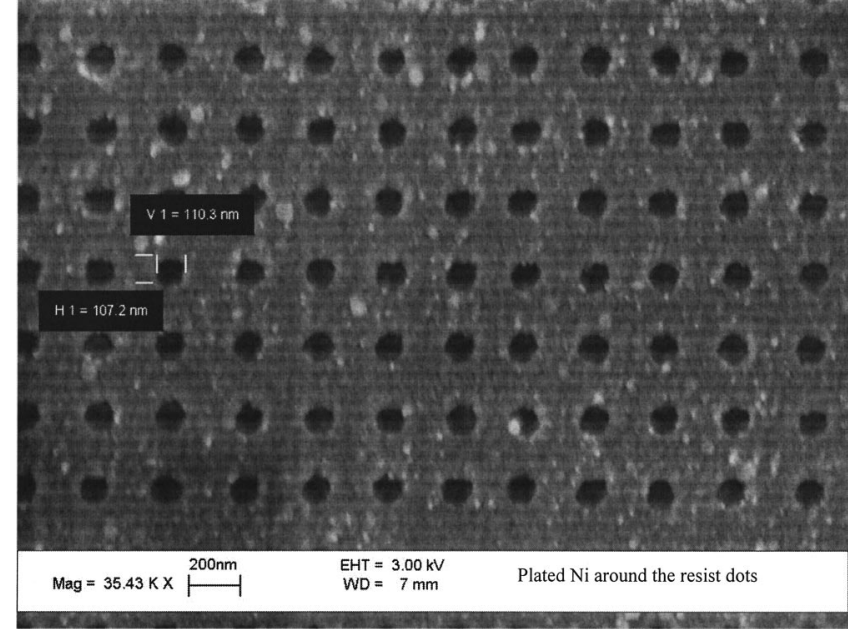

FIG. 3. SEM of $25 \mathrm{~nm}$ thick plated Ni around arrays of dots in the resist.

rounded by $25 \mathrm{~nm}$ thick plated $\mathrm{Ni}$ that has been used as a hard etch mask for these holes. As it can be seen from Fig. 3 the Ni surface is smooth free from voids with little defects and certainly conformed precisely to the e-beam written resist structures. The average size and pitch of the holes were 100 and $270 \mathrm{~nm}$, respectively. The test pattern holes, after etching through the $\mathrm{Si}_{3} \mathrm{~N}_{4}$ substrate, have an average diameter of $100 \mathrm{~nm}$ with $3 \sigma=21 \mathrm{~nm}$. Figure 4 shows a SEM high resolution image of holes etched in the $\mathrm{Si}_{3} \mathrm{~N}_{4}$ underlayer after stripping of the plated $\mathrm{Ni}$ and the $\mathrm{Ag}$ seed layer. It is important to mention that the holes have the same size after stripping the Ni and Ag. This experiment confirmed the excellent performance of the electroplated nickel as a hard-etch mask. The superior etching resistance of $\mathrm{Ni}$, in addition to its mirrorlike surface and the ability to plate very thin films, makes this technique very suitable to be employed for those applications that are demanding in terms of accuracy and etch depth. It is important to note that no dose correction or

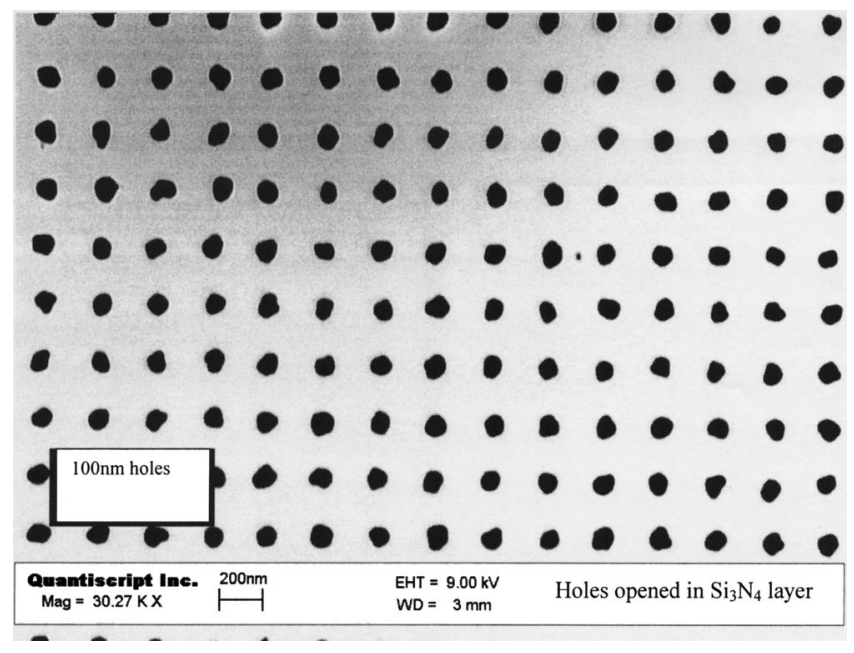

FIG. 4. Array of $110 \mathrm{~nm}$ holes after dry etch of $\mathrm{Ag}$ seed layer and the subsequent $\mathrm{Si}_{3} \mathrm{~N}_{4}$ layer, followed by removal or the etch mask and the seed layer. proximity pattern correction was used during exposure by the electron-beam lithography and that a uniform dose was used for all the dots. By combining a dry e-beam resist such as QSR-5 and electroplating, it is possible to invert the tonality of any pattern on any substrate such as back patterning of membranes, ${ }^{20}$ flexible polymers or any relief structure. ${ }^{21}$ This is not possible using conventional electron sensitive resists since spin-coating does not produce a uniform thickness layer on very high relief structures.

\section{CONCLUSION}

In conclusion, holes array of $100 \mathrm{~nm}$ diam $270 \mathrm{~nm}$ pitch has been fabricated using a dry e-beam resist, e-beam lithography, electroplating, and conventional RIE. It has been established in the present work that a nickel film of $25 \mathrm{~nm}$ thickness free from voids or defects is deposited using pulse reversal plating. It should also be pointed out that a plating rate as low as $0.2 \mathrm{~nm} / \mathrm{s}$ is achieved in this work which in turn makes very accurate control of the deposit thickness an easy task to achieve by accurate control of the plating parameters. The process can be applied to invert any pattern on any surface since it does not require any spin coating. More work is in process to enhance the resolution limit of the dry resist that is being used in this study. Nevertheless, the current results demonstrate the compatibility of this fabrication method with large arrays of holes for optoelectronic and microelectronics applications.

${ }^{1}$ M. Nakao, S. Oku, H. Tanaka, Y. Shibata, A. Yokoo, T. Tamamura, and H. Masuda, Opt. Quantum Electron. 34, 183 (2002).

${ }^{2}$ T. Ebbesen, H. Lezec, H. Ghaemi, T. Thio, and P. Wolff, Nature (London) 391, 667 (1998).

${ }^{3}$ A. Zeid and H. Baudrand, IEEE Trans. Microwave Theory Tech. 50, 1198 (2002).

${ }^{4}$ X. D. Huang, L.-R. Bao, X. Cheng, L. J. Guo, S. W. Pang, and A. F. Yee, J. Vac. Sci. Technol. B 20, 2872 (2002).

${ }^{5}$ H. Masuda, K. Kanezawa, and K. Nishio, Chem. Lett. 31, 1218 (2002)

${ }^{6}$ A. Fernandez, J. Y. Decker, S. M. Herman, D. W. Phillion, D. W. Sweeney, and M. D. Perry, J. Vac. Sci. Technol. B 15, 2439 (1997)

${ }^{7}$ X. Chen, S. H. Zaidi, S. R. J. Brueck, and D. J. Devine, J. Vac. Sci. Technol. B 14, 3339 (1996).

${ }^{8}$ T. Terasawa, N. Hasegawa, H. Fukuda, and S. Katagiri, Jpn. J. Appl. Phys., Part 1 30, 2991 (1991).

${ }^{9}$ S. Nakao, A. Nakae, A. Yamaguchi, K. Tsujita, and W. Wakamiya, Jpn. J. Appl. Phys., Part 1 38, 2686 (1999).

${ }^{10}$ M. M. Alkaisi, R. J. Blaikie, and S. J. McNab, Microelectron. Eng. 57-58, 367 (2001).

${ }^{11}$ S. Babin and H. W. P. Koops, J. Vac. Sci. Technol. B 14, 3860 (1996).

${ }^{12}$ V. Foglietti, E. Cianci, and G. Giannini, Microelectron. Eng. 57-58, 807 (2001).

${ }^{13}$ S. Babin, M. Weber, and H. W. P. Koops, J. Vac. Sci. Technol. B 14, 4076 (1996).

${ }^{14}$ S. Morita et al., J. Appl. Phys. 51, 3938 (1980).

${ }^{15}$ F. O. Fong, H. C. Kuo, J. C. Wolfe, and J. N. Randall, J. Vac. Sci. Technol. B 6, 357 (1988)

${ }^{16}$ E. Lavallée, J. Beauvais, D. Drouin, and M. Cloutier, U.S. patent pending. 
${ }^{17}$ J. Spector, C. J. Jacobsen, and D. M. Tennant, J. Vac. Sci. Technol. B 15, 2872 (1997)

${ }^{18}$ D. Weiss, M. Peuker, and G. Schneider, Appl. Phys. Lett. 72, 1805 (1998).

${ }^{19}$ G. Schneider, T. Schliebe, and H. Aschoff, J. Vac. Sci. Technol. B 13,
2809 (1995).

${ }^{20}$ Y. Awad, E. Lavallee, J. Beauvais, D. Drouin, Pan Yang, D. Turcotte, and Lau Kien Mun, J. Vac. Sci. Technol. B 20, 3040 (2002).

${ }^{21}$ L.-R. Bao, X. D. Huang, L. J. Guo, S. W. Pang, and A. F. Yee, J. Vac. Sci. Technol. B 20, 2881 (2002). 